\title{
Contact Totally Umbilical Submanifolds of a Sasakian Space Form $(*)$.
}

\author{
IKUo IshihaRa - Masahtro Kor (Shinjuku-ku, Tokyo)
}

Summary. - We define a notion of contaot totally umbilical submanifolds of Sasalian space forms corresponds to those of totally umbilical submanifolds of complex space forms. We study a contact totally umbilical submanifold It of a Sasakian space form $\bar{M}(c)(c \neq-3)$ and prove that $M$ is an invariant submanifold or an anti-invariant submanifold. Furthermore we study a submanifold $M$ with parallel second fundamental form of a Sasatian space form $\bar{M}(c)(o \neq 1)$ and prove that $M$ is invariant or anti-invariant.

\section{Introduction.}

Let $\bar{M}$ be a $(2 m+1)$-dimensional Sasakian manifold with strueture tensors $(\varphi, \xi, \eta, \bar{g})$. Then the structure tensors of $\bar{M}$ satisfy

$$
\begin{aligned}
& \varphi^{2} X=-X+\eta(X) \xi, \quad \varphi \xi=0, \quad \eta(\varphi X)=0, \quad \eta(\xi)=1, \\
& \bar{g}(\varphi X, \varphi Y)=\bar{g}(X, Y)-\eta(X) \eta(Y), \quad \eta(X)=\bar{g}(X, \xi),
\end{aligned}
$$

for any vector fields $X$ and $Y$ on $\bar{M}$. Let $\bar{\nabla}$ be the operator of covariant differentiation with respect to $\bar{g}$. Then we have

$$
\bar{\nabla}_{X} \xi=\varphi X, \quad\left(\bar{\nabla}_{X} \varphi\right) X=\bar{R}(X, \xi) Y=-\bar{g}(X, Y) \xi+\eta(Y) X
$$

for any vector fields $X$ and $Y$ on $\bar{M}$, where $\bar{R}$ denotes the Riemannian curvature tensor of $\bar{M}$.

Let $M$ be an $(n+1)$-dimensional submanifold tangent to the structure vector field $\xi$ of $\bar{M}$. We denote by $g$ the induced metric tensor field on $M$. Let $\nabla$ be the operator of covariant differentiation with respect to $g$. Then the Gauss and Weingarten formulas are given by

$$
\bar{\nabla}_{X} Y=\nabla_{X} Y+B(X, Y) \quad \text { and } \quad \bar{\nabla}_{X} N=-A_{N}(X)+D_{X} N
$$

for any vector fields $X, Y$ tangent to $M$ and any vector field $N$ normal to $M$, where $D$ is the operator of covariant differentiation with respect to the linear connection induced in the normal bundle $T(M)^{\perp}$. Both $A$ and $B$ are called the second fundamental

(*) Entrata in Redazione il 7 settembre 1976. 
forms of $M$ and they satisfy $\bar{g}(B(X, Y), N)=g\left(A_{N}(X), Y\right)$. A normal vector field $N$ is said to be parallel if $D_{X} N=0$ for any vector field $X$ tangent to $M$. The mean curvature vector $\mu$ of $M$ in $\bar{M}$ is defined as $\mu=(\operatorname{Tr} B) /(n+1)$, where $\operatorname{Tr} B=\sum_{i=1}^{n+1} B\left(e_{i}, e_{i}\right)$ for an orthonormal frame $\left\{e_{i}\right\}$ of $M$. If the second fundamental form $B$ of $M$ is of the form $B(X, Y)=g(X, Y) \mu$, then $M$ is said to be totally umbilical. In particular, if the second fundamental form $B$ vanishes indentically, $M$ is said to be totally geodesic. If $\mu=0$, then $M$ is said to be minimal. If the second fundamental form $B$ of $M$ is of the form

$$
B(X, Y)=[g(X, Y)-\eta(X) \eta(Y)] \alpha+\eta(X) B(Y, \xi)+\eta(Y) B(X, \xi)
$$

for any vector fields $X$ and $Y$ tangent to $M$, where $\alpha$ denotes a normal vector field to $M$, then $M$ is said to be contact totally umbilical. The notion of contact totally umbilical submanifolds of Sasakian manifolds corresponds to those of totally umbilical submanifolds of Kählerian manifolds (See [8]).

A submanifold $M$ of a Sasakian manifold $\bar{M}$ is said to be invariant if the structure vector field $\xi$ of $\bar{M}$ is tangent to $M$ and each tangent space $T_{x}(M)$ of $M$ is mapped into itself under the action of $\varphi$, i.e., $\varphi T_{\infty}(M) \subset T_{x}(M)$ for all $x \in M$. On the other hand, if each tangent space of $M$ is mapped into the normal space of $M$ by $\varphi$, i.e., $\varphi T_{x}(M) \subset T_{x}(M)^{\perp}$ for all $x \in M, M$ is said to be anti-invariant in $\bar{M}$.

A Sasakian manifold $\bar{M}$ of constant $\varphi$-sectional eurvature $c$ is called a Sasakian space form, denote it by $\bar{M}(c)$, and a Riemannian manifold of constant curvature is called a real space form.

CARTAN [2] proved that an $n$-dimensional totally umbilical submanifold of a euclidean $m$-space is either an $n$-plane or an $n$-sphere. Furthermore CHEN and OGIUE [4] proved a corresponding result for totally umbilical submanifolds of $a$ Kählerian manifold of constant holomorphic sectional curvature $c \neq 0$.

The purpose of the present paper is to prove the following

THEOREM 1. - Let $M$ be an $(n+1)$-dimensional submanifold tangent to the structure vector field $\xi$ of a $(2 m+1)$-dimensional Sasakian space form $\bar{M}^{2 m+1}(c)$ of constant $\varphi$-sectional curvature $c \neq 1$. If the second fundamental form $B$ of $M$ is parallel, then $M$ is one of the following submanifolds:

(a) a Sasakian space form immersed in $\bar{M}^{2 m+1}(c)$ as an invariant and totally geodesic submanifold, or

(b) an anti-invariant submanifold immersed in $\bar{M}^{2 n+1}(e)$ in $\bar{M}^{2 m+1}(c)$, where $\bar{M}^{2 n+1}(c)$ is an invariant and totally geodesic submanifold of $\bar{M}^{2 m+1}(c)$ of constant $\varphi$-sectional curvature $c \neq 1$.

THEOREM 2. - Let $M$ be an $(n+1)$-dimensional $(n \geqq 2)$ contact totally umbilical submanifold tangent to the structure vector field $\xi$ of a $(2 m+1)$-dimensional Sasakian 
space form $\bar{M}^{2 m+1}(c)$ of constant $\varphi$-sectional eurvature $c \neq-3$. Then $M$ is one of the following submanifolds:

(a) a Sasakian space form immersed in $\bar{M}^{2 m+1}(c)$ as an invariant and totally geodesic submanifold, or

(b) an anti-invariant submanifold immersed in $\bar{M}^{2 m+1}(c)$ as locally a Riemannian direct product $M^{n} \times M^{1}$, where $M^{n}$ is a real space form, a totally geodesic hypersurface of $M$ and a totally geodesic submanifold of $\bar{M}^{2 m+1}(c)$ and $M^{1}$ is a 1-dimensional space generated by $\xi$, or

(c) an anti-invariant submanifold immersed in $\bar{M}^{2 m+1}(c)$ as locally a Riemannian direct product $M^{n} \times M^{1}$, where $M^{n}$ is a real space form and a totally geodesic hypersurface of $M$ with nonzero parallel mean curvature vector in $\bar{M}^{2 m+1}(c)$ and $M^{1}$ is a 1.dimensional space generated by $\xi$.

Case (b) occurs only when $m \geqq n$ and case (c) occurs only when $m>n$.

\section{1. - Preliminaries.}

Let $M$ be an $(n+1)$-dimensional submanifold tangent to the structure vector field $\xi$ of a $(2 m+1)$-dimensional Sasakian manifold $\bar{M}$. For the second fundamental form $B$ of $M$ we define its covariant derivative, denoted by $\nabla_{X} B$, to be

$$
\left(\nabla_{X} B\right)(Y, Z)=D_{X}(B(Y, Z))-B\left(\nabla_{X} Y, Z\right)-B\left(Y, \nabla_{X} Z\right)
$$

for any vector fields $X, Y$ and $Z$ tangent to $M$. If $\nabla_{X} B=0$ for any vector field $X$ tangent to $M$, then the second fundamental form $B$ is said to be parallel. We denote by $R$ the Riemannian curvature tensor of $M$ and by $R^{\perp}$ the curvature tensor of the normal bundle $T(M)^{\perp}$, which is defined to be $R^{\perp}(X, Y)=\left[D_{X}, D_{Y}\right]-D_{[X, Y]}$. Then the equations of Gauss, Codazzi and Ricci are given respectively by

$$
\begin{aligned}
& \bar{g}(\bar{R}(X, Y) Z, W)=g(R(X, Y) Z, W)+\bar{g}(B(X, Z), B(Y, W)) \\
& -\bar{g}(B(X, W), B(\bar{X}, Z)) \\
& (\bar{R}(X, Y) Z)^{\perp}=\left(\nabla_{X} B\right)(Y, Z)-\left(\nabla_{Y} B\right)(X, Z)
\end{aligned}
$$

and

$$
\bar{g}(\bar{R}(X, Y) N, V)=\bar{g}\left(R^{\perp}(X, Y) N, V\right)-g\left(\left[A_{N}, A_{V}\right](X), Y\right)
$$

for any vector fields $X, Y, Z, W$ tangent to $M$ and any vector fields $N, V$ normal to $M$, where $\perp$ in (1.3) denotes the normal projection.

23 - Annali di Matematica 
A Sasakian manifold $\bar{M}$ is of constant $\varphi$-sectional curvature $e$ if and only if

$$
\begin{aligned}
\vec{R}(X, Y) Z & =\frac{1}{4}(c+3)[\bar{g}(Y, Z) X-\bar{g}(X, Z) Y] \\
& +\frac{1}{4}(c-1)[\eta(X) \eta(Z) Y-\eta(X) \eta(Z) X+\bar{g}(X, Z) \eta(Y) \xi-\bar{g}(Y, Z) \eta(X) \xi \\
& +\bar{g}(\varphi Y, Z) \varphi X-\bar{g}(\varphi X, Z) \varphi Y-2 \bar{g}(\varphi X, Y) \varphi Z]
\end{aligned}
$$

for any vector fields $X, Y$ and $Z$ on $\bar{M}$.

Now we state some lemmas for later use.

LEMMA 1 ([16]). - Let $M$ be an invariant submanifold of a Sasakian manifold $\bar{M}$. Then $M$ is totally geodesic if and only if the second fundamental form $B$ of $M$ is parallel.

LEMMA $2([14])$. - Let $M$ be an $(n+1)$-dimensional anti-invariant submanifold tangent to the structure vector field $\xi$ of a $(2 m+1)$-dimensional Sasakian manifold $\bar{M}$. Then the structure vector field $\xi$ is parallel on $M$ and $M$ is locally a Riemannian direct product $M^{n} \times M^{1}$, where $M^{n}$ is a totally geodesic hypersurface of $M$ and $M^{1}$ is a 1-dimensional space generated by $\xi$.

LEMMA 3 ([14]). - Let $M$ be an anti-invariant submanifold of a Sasakian manifold $\bar{M}$. If $\operatorname{dim} M \geqq 2$, then $M$ is not totally umbilical.

Let $M$ be an $(n+1)$-dimensional submanifold tangent to the structure vector field $\xi$ of a Sasakian manifold $\bar{M}$. Then the Gauss formula implies that

$$
\varphi X=\bar{\nabla}_{X} \xi=\nabla_{X} \xi+B(X, \xi)
$$

from which we have

$$
(\varphi X)^{T}=\nabla_{X} \xi \quad \text { and } \quad(\varphi X)^{\perp}=B(X, \xi),
$$

where $(\varphi X)^{T}$ and $(\varphi X)^{\perp}$ denote the tangential and normal parts of $\varphi X$ respectively. Since $\varphi \xi=0$, the second equation of (1.6) implies that

$$
B(\xi, \xi)=0
$$

If we assume that $M$ is totally umbilical, then we have $B(X, Y)=g(X, Y) \mu$, which implies that $0=B(\xi, \xi)=\mu$. That is, $M$ is minimal in $\bar{M}$ and hence $M$ is totally geodesic. Therefore we have

Proposition 1. - Let $M$ be a submanifold tangent to the structure vector field $\xi$ of a Sasakian manifold $\bar{M}$. If $M$ is totally umbilical, then $M$ is totally geodesic. 
IKUO IshIHaRa - MasaHtro Kox: Contact totally umbilical submanifolds, ete. 355

REMARK. - If $M$ is totally geodesic, then the second equation of (1.6) shows that $(\varphi X)^{\perp}=\mathbf{0}$. Therefore $\phi X$ is tangent to $M$ and hence $M$ is an invariant submanifold and a Sasakian manifold (See [12]).

Now we define a tensor field $T$ on $\bar{M}$ by setting

$$
T(X, Y) Z=\bar{R}(X, Y) Z+\bar{g}(\varphi Y, Z) \varphi X-\bar{g}(\varphi X, Z) \varphi Y-2 \bar{g}(\varphi X, Y) \varphi Z
$$

for any vector fields $X, Y$ and $Z$ on $\bar{M}$.

Let $M$ be a submanifold tangent to the structure vector field $\xi$ of $\bar{M}$. If each tangent space $T_{x}(M)$ of $M$ satisfies $(T(X, Y)) T_{x}(M) \subset T_{x}(M)$ for any vector fields $X$ and $Y$ tangent to $M$, then $M$ is said to be $T$-invariant. The notion of $T$-invariant corresponds to those of curvature-invariant of submanifolds in Kählerian manifolds in $[4]$ (See (5.4) in [9]).

Proposition 2. - Let $M$ be a submanifold tangent to the structure vector field $\xi$ of a Sasakian space form $\bar{M}(c)(c \neq-3)$. Then $M$ is $T$-invariant if and only if $M$ is invariant or anti-invariant.

Proof. - From (1.5) and (1.8) we have

$$
\begin{aligned}
& T(X, Y) Z \\
= & \frac{1}{4}(c+3)[\bar{g}(Y, Z) X-\bar{g}(X, Z) Y+\bar{g}(\varphi Y, Z) \varphi X-\bar{g}(\varphi X, Z) \varphi Y-2 \bar{g}(\varphi X, Y) \varphi Z] \\
+ & \frac{1}{4}(c-1)[\eta(X) \eta(Z) Y-\eta(Y) \eta(Z) X-g(X, Z) \eta(Y) \xi-g(Y, Z) \eta(X) \xi]
\end{aligned}
$$

for any vector fields $X, Y$ and $Z$ tangent to $M$. If $M$ is invariant or anti-invariant, then (1.9) implies that $M$ is $T$-invariant. Conversely if we assume that $M$ is $T$-invariant, then $M$ is invariant or anti-invariant. In fact, taking vectors $X, Y$ and $Z$ tangent to $M$ and orthogonal to $\xi$ and putting $X=Z$, we have

$$
T(X, Y) X=\frac{1}{4}(c+3)[\bar{g}(Y, X) X-\bar{g}(X, X) Y+3 \bar{g}(X, \varphi Y) \varphi X]
$$

Since $T(X, Y) X \in T_{x}(M)$, we have $\bar{g}(X, \varphi Y) \varphi X \in T_{x}(M)$ so that either $\varphi X \in T_{x}(M)$ or $g(X, \varphi Y)=0$. This implies that either $\varphi\left(T_{x}(M)\right) \subset T_{x}(M)$ or $\varphi\left(T_{x}(M)\right) \subset T_{x}(M)^{\perp}$. Consequently $M$ is invariant or anti-invariant.

If each tangent space $T_{x}(M)$ of $M$ satisfies $(\bar{R}(X, Y)) T_{x}(M) \subset T_{x}(M)$ for all vector fields $X$ and $Y$ tangent to $M$, then $M$ is said to be $\bar{R}$-invariant.

Proposition 3. - Let $M$ be a submanifold tangent to the structure vector field $\xi$ of a Sasalian space form $\bar{M}(c)(c \neq 1)$. Then $M$ is $\bar{R}$-invariant if and only if $M$ is invariant or anti-invariant. 
Proof. - If $M$ is invariant or anti-invariant, by (1.5) we easily see that $M$ is $\bar{R}$-invariant. Let us assume that $M$ is $\bar{R}$-invariant. Then (1.5) implies that

$$
\vec{g}(\varphi Y, Z) \varphi X-\bar{g}(\varphi X, Z) \varphi Y-2 \bar{g}(\varphi X, Y) \varphi Z
$$

is tangent to $M$. Putting $X=Z$, we see that $\bar{g}(\varphi Y, X) \varphi X \in T_{x}(M)$. Therefore $M$ is invariant or anti-invariant.

\section{2. - Proof of Theorem 1 .}

Let $M$ be an $(n+1)$-dimensional submanifold tangent to the structure vector field $\xi$ of a Sasakian space form $\bar{M}^{2 m+1}(c)(c \neq 1)$ with parallel second fundamental form. Then the Codazzi equation (1.3) reduces to $(R(X, Y) Z)^{\perp}=0$ for any vector fields $X, Y$ and $Z$ tangent to $M$. Therefore $M$ is $\bar{R}$-invariant. Thus Proposition 3 implies that $M$ is invariant or anti-invariant. Let $M$ be an invariant submanifold. Then Lemma 1 proves $(a)$ of Theorem 1 . In the following we assume that $M$ is an anti-invariant submanifold of $\bar{M}^{2 m+1}(c)$. Then the normal space $T_{x}(M)^{\perp}$ is decomposed as

$$
T_{x}(M)^{\perp}=\varphi\left(T_{x}(M)\right) \oplus N_{x}(M)
$$

where $N_{x}(M)$ is the complement of $\varphi\left(T_{x}(M)\right)$ in $T_{x}(M)$. Then we see $\varphi\left(N_{x}(M)\right)$ $=N_{x}(M)$. Since $M$ is anti-invariant, by $(1.6)$, we have $p X=B(X, \xi)$. On the other hand, by (1.1) and Lemma 2, we have that $D_{X}(\varphi \bar{Y})=\varphi \nabla_{X} Y$ for any vector fields $X$ and $Y$ tangent to $M$. Using the Gauss and Weingarten formulas, we obtain

$$
\eta(Y) X-\bar{g}(X, Y) \xi+\varphi B(X, Y)+A_{\varphi Y}(X)=0 .
$$

Therefore we have

$$
\eta(Y) \varphi X-B(X, Y)+\varphi A_{\varphi Y}(X)=0 .
$$

From this, for any vector $N \in N_{x}(M)$, we have that $\bar{g}(B(X, Y), N)=0$. Thus we have

$$
A_{N}(X)=0 \quad \text { for } N \in N_{x}(M)
$$

Now our assertion $(b)$ of Theorem 1 reduces to the following

Proposition 4. - Let $M$ be an $(n+1)$-dimensional anti-invariant submanifold tangent to the structure vector field $\xi$ of a Sasakian space form $\bar{M}^{2 m+1}(c)$. If $A_{N}(X)=0$ for any vector $N \in N_{x}(M)$, then $M$ is immersed in $\bar{M}^{2 n+1}(c)$ and $\bar{M}^{2 m+1}(c)$, where $\bar{M}^{2 n+1}(c)$ is an invariant and totally geodesic submanifold of $\bar{M}^{2 m+1}(c)$. 
Proof. - First of all, we prove that $\varphi T(M)$ is parallel with respect to the normal connection, that is, for every local section $\varphi Y$ in $\varphi T(M I), D_{X} \varphi Y$ is also a local section in $\varphi T(M)$.

From the Gauss and Weingarten formulas we have

$$
\begin{aligned}
D_{X} \varphi Y & =\bar{\nabla}_{X} \varphi \bar{Y}+A_{\varphi Y}(X) \\
& =\left(\bar{\nabla}_{X} \varphi\right) Y+\varphi \bar{\nabla}_{X} Y+A_{\varphi Y}(X) \\
& =\eta(Y) X-\bar{g}(X, Y) \xi+\varphi \nabla_{\varphi_{X}} Y+\varphi B(X, Y)+A_{\varphi Y}(X) .
\end{aligned}
$$

Since $A_{N}=0$ for any $N \in N_{x}(M)$, we have

$$
\begin{aligned}
\bar{g}\left(D_{X} \varphi Y, N\right) & =\bar{g}(\varphi B(X, Y), N) \\
& =-\bar{g}(B(X, Y), \varphi N)=-g\left(A_{\varphi_{N}}(X), Y\right)=0
\end{aligned}
$$

because of $\varphi N$ is also in $N_{X}(M)$. Consequently, $\varphi T(M)$ is parallel with respect to the normal connection. Moreover $\varphi T(M)$ is of constant dimension on $M$. Therefore, from the fundamental theorem in submanifold theory, there exists a totally geodesic Sasakian submanifold $\bar{M}^{2 n+1}(c)$ in $\bar{M}^{2 m+1}(c)$, where $M$ is immersed in $\bar{M}^{2 n+1}(c)$ as an anti-invariant submanifold. This completes our assertion.

EXAMIPLE 1. - Let $E^{2 n+1}$ be a euclidean space with cartesian coordinates $\left(x^{1}, \ldots, x^{n}, y^{1}, \ldots, y^{n}, z\right)$. Then a Sasakian structure on $E^{2 n+1}$ is defined by $\varphi, \xi, \eta$ and $\vec{g}$ such that

$$
\begin{aligned}
& \xi=(0, \ldots, 0,2), \quad 2 \eta=\left(-y^{1}, \ldots,-y^{n}, 0, \ldots, 0,1\right) \\
&\left(\bar{g}_{A B}\right)=\left(\begin{array}{ccc}
\frac{1}{4}\left(\delta_{i j}+y^{i} y^{j}\right) & 0 & -\frac{1}{4} y^{i} \\
0 & \frac{1}{4} \delta_{i j} & 0 \\
-\frac{1}{4} y^{j} & 0 & \frac{1}{4}
\end{array}\right), \\
&\left(\varphi_{B}^{A}\right)=\left(\begin{array}{ccc}
0 & \delta_{j}^{i} & 0 \\
-\delta_{j}^{i} & 0 & 0 \\
0 & y^{j} & 0
\end{array}\right) .
\end{aligned}
$$

Then $E^{2 n+1}$ is of constant $\varphi$-sectional curvature -3 and denoted by $E^{2 n+1}(-3)$ (ef. [10] and [11]).

Now we can consider the following natural imbedding of $E^{n+1}$ into $E^{2 n+1}(-3)$ :

$$
\left(x^{\mathbf{1}}, \ldots, x^{n}, z\right) \mapsto\left(x^{1}, \ldots, x^{n}, 0, \ldots, 0, z\right) \text {. }
$$


Then the second fundamental forms of $E^{n+1}$ in $E^{2 n+1}(-3)$ are given by

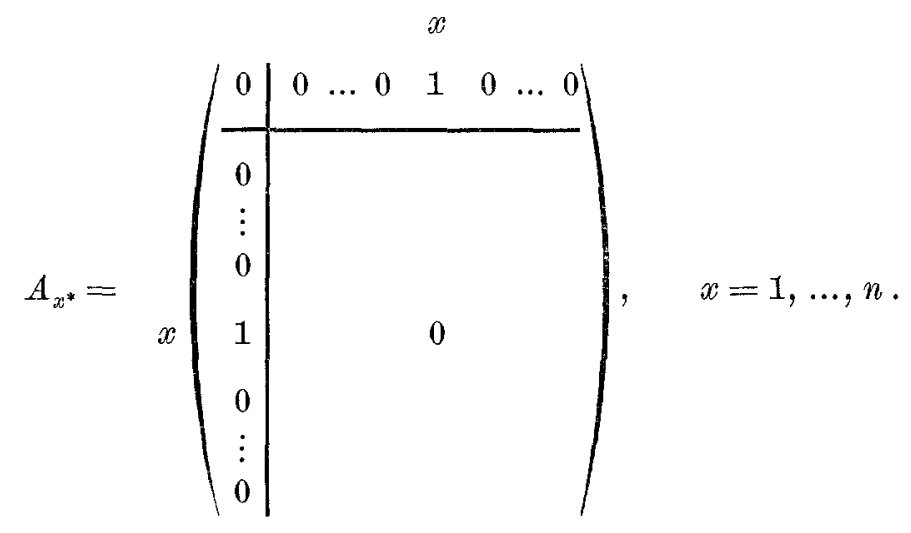

Thus the second fundamental form of $E^{n+1}$ is parallel and $E^{n+1}$ is obviously antiinvariant in $E^{2 n+1}(-3)$. Since $E^{2 n+1}(-3)$ is a totally geodesic and invariant submanifold of $E^{2 m+1}(-3)(m>n), E^{n+1}$ is an anti-invariant submanifold of $E^{2 m+1}(-3)$ with parallel second fundamental form.

Let $S^{1}\left(r_{i}\right)=\left\{z_{i} \in C:\left|z_{i}\right|^{2}=r_{i}^{2}\right\}$ be circles of radius $r_{i}, i=1, \ldots, n$. Now we consider

$$
M^{n+1}=S^{1}\left(r_{1}\right) \times \ldots \times S^{1}\left(r_{n}\right) \times E^{1}
$$

Then we can consider an imbedding $M^{n+1}$ into $E^{2 n+1}(-3)$ given by

$$
\left(r_{1} \cos u^{1}, \ldots, r_{n} \cos u^{n}, r_{1} \sin u^{1}, \ldots, r_{n} \sin u^{n}, z\right)
$$

Then $M^{n+1}$ is an anti-invariant submanifold of $E^{2 n+1}(-3)$ and hence of $E^{2 m+1}(-3)$. The second fundamental forms of $M^{n+1}$ in $E^{2 n+1}(-3)$ are given by

$$
A_{x^{*}}=\left(\begin{array}{c|ccccccc}
0 & 0 & \ldots & 0 & 1 & 0 & \ldots & 0 \\
\hline 0 & 0 & & & & & \\
\vdots & & \ddots & & & & \\
0 & & 0 & & & \\
1 & & & & \lambda_{x} & & \\
0 & & & & 0 & \\
\vdots & & & & & \ddots & \\
0 & & & & & & 0
\end{array}\right), \quad x=1, \ldots, n .
$$

And the second fundamental form of $M^{n+1}$ is parallel. 
REMARK. - Let $\delta^{2 n+1}$ be a $(2 n+1)$-dimensional unit sphere, i.e., $\$^{2 n+1}=$ $=\left\{z \in C^{n+1}:|z|=1\right\}$. For any point $z \in S^{2 n+1}$, put $\xi=J z$, where $J$ is the almost complex structure of $C^{n+1}$. We consider the orthogonal projection

$$
\pi: T_{z}\left(C^{n+1}\right) \rightarrow T_{z}\left(S^{2 n+1}\right) .
$$

Putting $\varphi=\pi \circ J$, we have a Sasakian structure $(\varphi, \xi, \eta, \bar{g})$ on $\mathcal{g}^{2 n+1}$, where $\eta$ is a 1 -form dual to $\xi$ and $\bar{g}$ the standard metric tensor field on $S^{9 n+1}$. We see that $\$^{2 n+1}$ is of constant $\varphi$-sectional curvature 1 , that is, of constant curvature 1 (cf. [10] and [11]).

Now we consider the Clifford hypersurface $M_{p, q}$ defined by

$$
M_{p, q}=S^{2 p+1}\left(\sqrt{\frac{p}{2 n}}\right) \times S^{2 q+1}\left(\sqrt{\frac{q}{2 n}}\right), \quad p+q=n-1 .
$$

Then $M_{p, q}$ is a minimal hypersurface of $S^{2 n+1}$ tangent to the structure vector field $\xi$ of $S^{2 n+1}$ and $M_{p, q}$ has the parallel second fundamental form. But $M_{p, q}$ is neither invariant nor anti-invariant in $S^{2 n+1}$. Therefore the assumption in Theorem 1 on the $\varphi$-sectional curvature $c \neq 1$ of the ambient manifold $\bar{M}^{2 m+1}(c)$ is essential.

On the other hand, there exists an example of anti-invariant submanifold with parallel second fundamental form of $\bar{M}^{2 m+1}(1)$. Indeed, if we consider

$$
M^{n+1}=S^{1}\left(r_{1}\right) \times \ldots \times S^{1}\left(r_{n+1}\right), \quad r_{1}^{2}+\ldots+r_{n+1}^{2}=1
$$

in $S^{2 n+1}$, then $M^{n+1}$ is an anti-invariant submanifold of $S^{2 n+1}$ with parallel second fundamental form (See [14]).

\section{3. - Proof of Theorem 2.}

Let $M$ be an $(n+1)$-dimensional contact totally umbilical submanifold tangent to the structure vector field $\xi$ of a Sasalkian space form $\bar{M}^{2 m+1}(c)(c \neq-3)$. Then we have

$$
B(X, Y)=[g(X, Y)-\eta(X) \eta(Y)] H+\eta(X) B(Y, \xi)+\eta(Y) B(X, \xi)
$$

for any vector fields $X$ and $Y$ tangent to $M$, where $H$ is given by $H=(\operatorname{Tr} B) / n$. From (1.1) and (3.1) we have

$$
\begin{aligned}
\left(\nabla_{X} B\right)(Y, Z) & =[g(Y, Z)-\eta(Y) \eta(Z)] D_{X} H+\bar{g}(\varphi X, X) B(Z, \xi) \\
& +\bar{g}(\varphi X, Z) B(Y, \xi)-\bar{g}(\varphi X, Y) \eta(Z) H-\bar{g}(\varphi X, Z) \eta(Y) H \\
& +\eta(Y) B\left(Z, \nabla_{X} \xi\right)+\eta(Z) B\left(Y, \nabla_{X} \xi\right) \\
& +\eta(Y)\left(\nabla_{X} B\right)(Z, \xi)+\eta(Z)\left(\nabla_{Y} B\right)(Y, \xi) .
\end{aligned}
$$


IKUO IsHihara - MasaHIRo Kon: Contaet totally umbilical submanifolds, ete.

If $X, Y$ and $Z$ are orthogonal to $\xi$, using (1.6), we have

$$
\left(\nabla_{X} B\right)(Y, Z)=g(Y, Z) D_{X} H+\bar{g}(\varphi X, Y)(\varphi Z)^{\perp}+\bar{g}(\varphi X, Z)(\varphi Y)^{\perp}
$$

From the Codazzi equation (1.3) and (3.2) we obtain

$$
\begin{aligned}
(\bar{R}(X, Y) Z)^{\perp}=g(Y, Z) D_{X} H-g(X, Z) D_{Y} H & +\bar{g}(\varphi X, Z)(\varphi Y)^{\perp} \\
& +\bar{g}(\varphi Z, X)(\varphi X)^{\perp}-2 \bar{g}(\varphi Y, X)(\varphi Z)^{\perp}
\end{aligned}
$$

for any vector fields $X, Y$ and $Z$ orthogonal to $\xi$ on $M$. If $n \geqq 3$, for any vector field $X$ orthogonal to $\xi$ on $M$, we can choose a unit vector field $Y$ tangent to $M$ which is orthogonal to $X, \varphi X$ and $\xi$. For such a choice it follows from (3.3) that

$$
(\bar{R}(X, Y) Y)^{\perp}=D_{X} H
$$

On the other hand, (1.5) implies that $(\bar{R}(X, Y) Y)^{\perp}=0$. Therefore we have

$$
D_{x} H=0
$$

for any vector field $X$ tangent to $M$ and orthogonal to $\xi$. From (3.3) and (3.5) we have

$$
(\bar{R}(X, Y) Z)^{\perp}=\bar{g}(\varphi X, Z)(\varphi Y)^{\perp}+\bar{g}(\varphi Z, Y)(\varphi X)^{\perp}-2 \bar{g}(\varphi X, X)(\varphi Z)^{\perp}
$$

for any tangent vector fields $X, Y$ and $Z$ orthogonal to $\xi$ on $M$. Substituting (3.6) into (1.8) and taking the normal part, we have

$$
(T(X, Y) Z)^{\perp}=0 \text {. }
$$

Since $T(X, \xi) Z=0$ for any vector fields $X$ and $Y$ tangent to $M,(3.7)$ shows that $(T(X, Y) Z)^{\perp}=0$ for any vector fields $X, Y$ and $Z$ tangent to $M$, which means that $M$ is $T$-invariant.

If $n=2$, put $M=M_{1} \cup M_{2}$, where $M_{1}=\left\{x \in M: \varphi\left(T_{x}(M)\right) \neq T_{x}(M)-\{\xi\}\right\}$ and $M_{2}=\left\{x \in M: \varphi\left(T_{x}(M)\right)=T_{x}(M)-\{\xi\}\right\}$. We can see that $M_{1}$ is an open submanifold of $M$, where the preceding argument is available so that (3.7) holds on $M_{1}$. Let $M_{2}^{\prime}$ be the set of all interior points of $M_{2}$. Then $M_{2}^{\prime}$ is an invariant submanifold of $\bar{M}$ so that $H=0$ on $M_{2}^{\prime}$ and hence (3.7) holds on $M_{2}^{\prime}$. Since (3.7) is a tensorial equation, it holds on $M$. Therefore $M$ is $T$-invariant when $n \geqq 2$. Thus, by Proposition 2, $M$ is invariant or anti-invariant.

If $M$ is an invariant submanifold of $\bar{M}^{2 m+1}(c)$, then $M$ is minimal (cf. [6] and [12]). Then we have $\operatorname{Tr} B=0$ and hence $H=0$. By the second equation of (1.6), we also have $B(X, \xi)=0$ for any vector field $X$ tangent to $M$. Therefore (3.1) implies 
IKUO ISHTHARA - MASAHIRO KON: Contact totally umbilical submanifolds, ete. 361

that $B(X, Y)=0$ and hence $M$ is totally geodesic. This completes the proof of $(a)$ of Theorem 2.

In the sequel, we consider the case that $M$ is an anti-invariant submanifold. Since $\varphi\left(T_{x}(M)\right) \subset T_{x}(M)^{\perp}$, we see that $m \geqq n$. Moreover, by Lemma $2, M$ is locally a Riemannian direct product $M^{n} \times M^{1}$, where $M^{n}$ is a totally geodesic hypersurface of $M$ and $M^{1}$ is a 1 -dimensional space generated by $\xi$. Since $B(\xi, \xi)=0$, the mean curvature vector of $M^{n}$ in $\bar{M}^{2 m+1}(c)$ is just $H$. Moreover, we have $B(X, Y)=g(X, Y) H$ for any vector fields $X$ and $Y$ on $M^{n}$, which means that $M^{n}$ is a totally umbilical submanifold of $\bar{M}^{2 m+1}(c)$. From this and the Gauss equation (1.2) we see that $M^{n}$ is a real space form of constant curvature $\frac{1}{4}(c+3)+\bar{g}(H, H)$. Moreover, the mean curvature vector $H$ of $M^{n}$ in $\bar{M}^{2 m+1}(e)$ is parallel with respect to the linear connection $D^{\prime}$ induced in the normal bundle of $M^{n}$ in $\bar{M}^{2 m+1}(c)$, which is proved by the following way.

First of all, by a straight foward computation, using the Gauss equation, we have (See [14])

$$
A_{\varphi X}(Y)=A_{\varphi Y}(X)
$$

for any vector fields $X$ and $Y$ tangent to $M^{n}$. Since $B(X, Y)=g(X, Y) H$, we have

$$
g\left(A_{\varphi Z}(X), Y\right)=\bar{g}(B(X, Y), \varphi Z)=\bar{g}(X, Y) g(H, \varphi Z)
$$

for any vector fields $X, Y$ and $Z$ tangent to $M^{n}$. From (3.8) and (3.9) we obtain

$$
g(X, Y) \bar{g}(H, \varphi Z)=g\left(A_{\varphi X}(Z), Y\right)=g(Y, Z) \bar{g}(H, \varphi X)
$$

For any vector field $Z$ tangent to $M^{n}$, we can choose a unit vector field $X$ tangent to $M^{n}$ which is orthogonal to $Z$ and putting $X=Y$ in (3.10), we have

$$
\ddot{g}(H, \varphi Z)=0 .
$$

Therefore the mean curvature vector $H$ is orthogonal to $\varphi\left(T_{x}(M)\right)$. Thus if $H \neq 0$, then $m>n$. On the other hand, by the Weingarten formula, we have

$$
-A_{H}(X)+D_{X} H=-A_{H}^{\prime}(X)+D_{X}^{\prime} H
$$

where $A^{\prime}$ denotes the second fundamental form of $M^{n}$ in $\bar{M}^{2 m+1}(c)$. Then (3.5) implies that

$$
-A_{H}(X)=-A_{z}^{\prime}(X)+D_{X}^{\prime} H .
$$

Thus we obtain that $\bar{g}\left(D_{X}^{\prime} H, N\right)=0$ for any vector field $N$ orthogonal to $M$. More- 
over we have

$$
\bar{g}\left(D_{x}^{\prime} H, \xi\right)=-g\left(A_{H}(X), \xi\right)=-\bar{g}(B(X, \xi), H)=-\bar{g}(\varphi X, H)=0
$$

by using (1.6) and (3.11). Consequently we have

$$
D_{x}^{\prime} H=0
$$

which means that the mean curvature vector $H$ of $M^{n}$ in $\bar{M}^{2 m+1}(c)$ is parallel. Therefore we have $(b)$ and $(c)$ of Theorem 2.

EXAMPLE 2. - Let $S^{2 m+1}$ be a unit sphere of dimension $2 m+1$ with standard Sasakian structure and let $C P^{m}$ be a complex projective space of real dimension $2 m$ of constant holomorphic sectional curvature 4. A real projective space $R P^{n}$ of dimension $n$ of constant curvature 1 is imbedded in $C P^{n}$ in $C P^{m}$ as an anti-invariant and totally geodesic submanifold (See[1]). Now we consider the following commutative diagram:

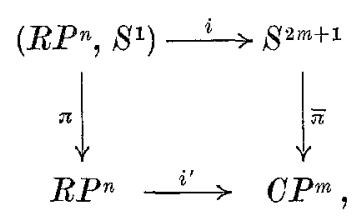

where $\left(R P^{n}, S^{1}\right)$ denotes a circle bundle over $R P^{n}$. Then $\left(R P^{n}, S^{1}\right)$ is an anti-invariant submanifold immersed in a $S^{2 n+1}$ in $S^{2 m+1}$ with parallel second fundamental form. Moreover, we see that $\left(R P^{n}, S^{1}\right)$ is a contact totally umbilical submanifold of $\mathbb{S}^{2 n+1}$ whose second fundamental forms are given by

$$
A_{x^{*}}=\left(\begin{array}{c|ccccccc}
0 & 0 & \ldots & 0 & 1 & 0 & \ldots & 0 \\
\hline 0 & & & & & \\
\vdots & & & & \\
0 & & & & \\
1 & & & & 0 & \\
0 & & & & &
\end{array}\right), x=1, \ldots, n
$$

Let $M^{r}$ be a totally umbilical submanifold of $R P^{n}$. Then $M^{r}$ is a totally umbilical submanifold of $O P^{n}$. Therefore the circle bundle $\left(M^{r}, S^{1}\right)$ over $M^{r}$ is a contact totally umbilical submanifold of $S^{2 n+1}$ (See [8]). The second fundamental forms $A_{3 *}$ of an 
anti-invariant contact totally umbilical submanifold $M^{n+1}$ of a Sasakian manifold $\bar{M}^{2 n+1}$ are given by

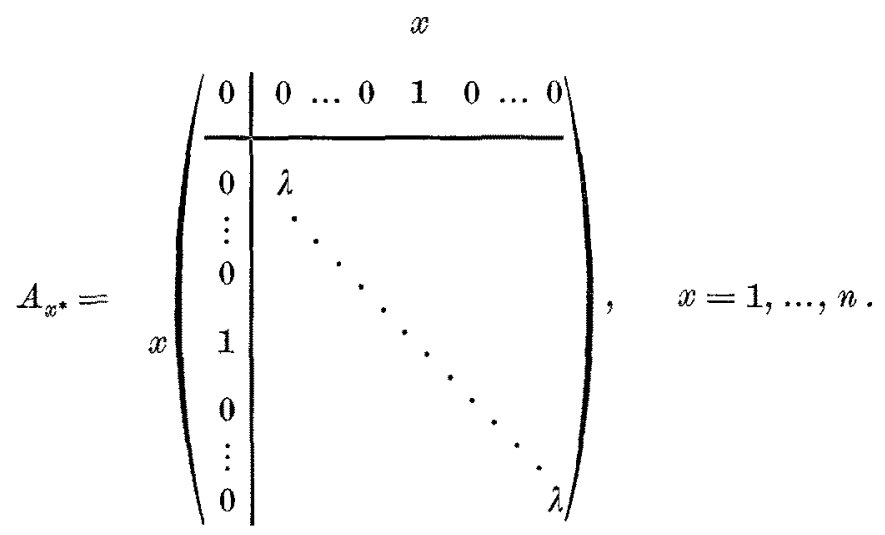

REMARK. - Let $E^{2 n+1}(-3)$ be a Sasakian space form of constant $\varphi$-sectional curvature -3 as in Example 1. Now we consider $S^{2 n-1} \times E^{1}=M^{2 n}$. Then $M^{2 n}$ is a submanifold of $E^{2 n+1}(-3)$ tangent to the structure vector field $\xi$ of $E^{2 n+1}(-3)$ and $M^{2 n}$ is contact totally umbilical. But $M^{2 n}$ is neither invariant nor anti-invariant in $E^{2 n+1}(-3)$. Therefore the assumption in theorem 2 on the $\varphi$-sectional curvature $e \neq-3$ of an ambient manifold $\bar{M}^{2 m+1}(e)$ is essential.

REMARK. - Let $M$ be an $(n+1)$-dimensional submanifold tangent to the structure vector field $\xi$ of a $(2 m+1)$-dimensional Sasakian manifold $\bar{M}$. If the second fundamental form $B$ of $M$ is of the form

$$
B(X, Y)=\eta(X) B(Y, \xi)+\eta(Y) B(X, \xi)
$$

for any vector field $X$ and $Y$ tangent to $M$, then $M$ is said to be contaet totally geodesic. The notion of contant totally geodesic submanifolds of Sasakian manifolds corresponds to those of totally geodesic submanifolds of Kählerian manifolds.

\section{REFERENCES}

[1] K. ABE, Applications of Riecati type differential equation to Riemannian manifolds with totally geodesie distribution, Tôhoku Math. Journ., 25 (1973), pp. 425-444.

[2] E. Cartan, Legon sur la géométrie des espaces de Riemann, Gauthier-Villars, Paris (1946).

[3] B. Y. Chen - K. Ogrun, On totally real submanifolds, Trans. Amer. Math. Soc., 193 (1974), pp. 257-266.

[4] B. Y. CHeN - K. OGrue, Two theorems on Kähler manifolds, Michigan Joum., 21 (1974), pp. $225-229$. 
[5] S. Kobayashi - K. Nomut, Foundations of differential geometry, vol. II, Wiley, Interscience Tracts, John Wiley, New York (1969).

[6] M. KoN, Invariant submanifolds of normal contact metrio manifolds, Ködai Math. Sem. Rep., 25 (1973), pp. 330-336.

[7] M. KoN, Totally real minimal submanifolds with parallel second fundamental form, Accademia Naz. dei Lincei, 57 (1974), pp. 187-189.

[8] M. KoN, Remarks on anti-invariant submanifolds of a Sasakian manifold, Tensor, N. S., 30 (1976), pp. $239-246$.

[9] K. OGrue, On fiberings of almost contact manifolds, Kōdai Math. Sem. Rep., 17 (1965), pp. 53-62.

[10] S. SASAKI, Almost contact manifolds, vol. I, Lecture Note in Tohoku University (1965).

[11] S. TaNNo, Sasakian manifolds with constant p.holomorphie sectional curvature, Tóhokn Math. Journ., 21 (1969), pp. $501-507$.

[12] K. YANO - S. IsHIHARA, Invariant submanifolds of almost contact manifolds, Kōai Math. Sem. Rep., 21 (1969), pp. 350-364.

[13] K. Yano - M. Kon, Totally real submanifolds of complex space forms, $I$, Tôhoku Math. Journ., 28 (1976), pp. 215-225.

[14] K. YaNo - M. KON, Anti-invariant submanifolds of Sasakian space forms I, Tôhoku Math. Journ., 29 (1977), pp. 9-23. 